\title{
Computed tomography versus plain radiography assessment of acetabular fracture reduction is more predictive for native hip survivorship
}

\author{
Diederik O. Verbeek ${ }^{1,2}$. Jelle P. van der List ${ }^{1}$. David L. Helfet ${ }^{1}$
}

Received: 17 October 2018 / Published online: 27 April 2019

(c) The Author(s) 2019

\begin{abstract}
Introduction Computed tomography (CT) is more accurate than plain pelvic radiography (PXR) for evaluating acetabular fracture reduction. As yet unknown is whether CT-based assessment is more predictive for clinical outcome. We determined the independent association between reduction quality according to both methods and native hip survivorship following acetabular fracture fixation.

Materials and methods Retrospectively, 220 acetabular fracture patients were reviewed. Reductions on PXR were graded as adequate or inadequate (0-1 mm or $>1 \mathrm{~mm}$ displacement) (Matta's criteria). For CT-based assessment, adequate reductions were defined as $<1 \mathrm{~mm}$ step and $<5 \mathrm{~mm}$ gap, and inadequate reductions as $\geq 1 \mathrm{~mm}$ step and/or $\geq 5 \mathrm{~mm}$ gap displacement. Predictive values and Kaplan-Meier hip survivorship curves were compared and risk factors for conversion to total hip arthroplasty (THA) were identified.

Results Mean follow-up was 8.9 years (SD 5.6, range 0.5-23.3 years), and 52 patients converted to THA (24\%). Adequate reductions according to $\mathrm{CT}$ versus PXR assessment were associated with higher predictive values for native hip survivorship (92\% vs. $82 \% ; p=0.043$ ). Inadequate reductions were equally predictive for conversion to THA (33\% for CT and $30 \%$ for PXR; $p=0.623)$. For both methods, survivorship curves of adequate versus inadequate reductions were significantly different $(p=0.030$ for PXR, $p<0.001$ for CT). Only age $\geq 50$ years $(p<0.001)$ and inadequate reductions as assessed on CT $(p=0.038)$ were found to be independent risk factors for conversion to THA. Reduction quality as assessed on PXR was not found to be independently predictive for this outcome $(p=0.585)$.

Conclusion Native hip survivorship is better predicted based on postoperative CT imaging as compared to PXR assessment. Predicting need for THA in patients with inadequate reductions based on both assessment methods remains challenging. While both PXR and CT-based methods are associated with hip survivorship, only an inadequate reduction according to CT assessment was an independent risk factor for conversion to THA.
\end{abstract}

Keywords Acetabular fracture · Hip survivorship · Total hip arthroplasty · Pelvic radiography · Computed tomography · Postoperative reduction

Diederik O. Verbeek

d.o.f.verbeek@erasmusmc.nl

1 Orthopaedic Trauma Service, Hospital for Special Surgery and New York Presbyterian Hospital, Weill Cornell Medicine, New York, NY, USA

2 Present Address: Trauma Research Unit, Department of Surgery, Erasmus MC, University Medical Center Rotterdam, Rotterdam, The Netherlands

\section{Introduction}

Native hip survivorship in patients who receive operative treatment for acetabular fractures is influenced by a variety of factors [1-3]. These include non-modifiable or predetermined factors, such as patient age, fracture impaction, posterior wall involvement, and damage to the femoral head. An important modifiable risk factor thought to be predictive for clinical outcomes is quality of acetabular fracture reduction [3, 4-7]. An anatomic postoperative reduction has widely been used as a (early) proxy for successful surgical treatment. 
Yet, a number of studies have noted that up to onethird of patients with an apparent anatomic reduction have poor clinical outcome following acetabular fracture surgery [6-8]. This seemingly contradictory finding may in part be attributed to the specific method used to assess reduction quality. In general, Matta's criteria based on plain pelvic radiography (PXR) are used for this purpose (Matta). However, it is currently well established that CT is superior in detecting residual fracture displacement and different criteria to grade accuracy of reduction may have to be applied for this modality [9-12].

While it is evident that CT is more accurate than PXR for evaluation of acetabular fracture reduction, it remains to be determined whether CT-based assessment using criteria specifically designed for this modality is also more predictive for clinical outcome. Therefore, the purpose of this study is to determine the (independent) association between reduction quality according to both methods and native hip survivorship following acetabular fracture fixation.

\section{Materials and methods}

\section{Study design}

Following Institutional Review Board approval, a search in our Orthopedic Trauma Service (OTS) registry was performed for all adult patients who underwent surgical fixation for an acute (within 3 weeks of injury) isolated acetabular fracture between the dates of January 1994 and June 2014. Surgical indications for open reduction and internal fixation (ORIF) of acetabular fractures were $\geq 2 \mathrm{~mm}$ fracture displacement within the weight-bearing dome, an incongruent hip joint, or an unstable posterior wall fracture. All surgeries were performed by the senior author. Within the first postoperative week, all patients routinely underwent PXR in three standard radiographic views (antero-posterior, iliac oblique, and obturator oblique) as well as pelvic CT imaging.

\section{Inclusion and exclusion criteria}

A total of 481 consecutive acetabular fracture patients were found to be eligible for inclusion. Patients were excluded if follow-up was less than 2 years; unless they converted to total hip arthroplasty (THA) in this interval (208 exclusions), if they had concomitant femoral head fractures (six exclusions) or pelvic ring fractures (two exclusions), or if postoperative PXR and/or CT imaging was not available for review (45 exclusions). This left 220 patients to be included in the study.

\section{Data collection}

Data were retrospectively collected from our prospective OTS registry and included patient demographics and fracture types, as classified by the senior author (***) using the Letournel acetabular fracture classification system $[8,11]$. Patients were routinely followed up in clinic at regular intervals (at 2 weeks and 1, 3, 6, and 12 months, and annually thereafter). In addition, all patients were contacted by mail and telephone to determine the most current status of their native hip (preserved native hip versus failed native hip with conversion to THA).

\section{Quality of reduction}

Postoperative PXRs were assessed for residual gap and/ or step displacement in the three standard views and the greatest measurement was used to grade quality of reduction according to Matta's system [6]. In accordance with prior studies, adequate (or anatomic) reductions with $0-1 \mathrm{~mm}$ of displacement were compared to inadequate (imperfect or poor) reductions with $>1 \mathrm{~mm}$ displacement $[2,3]$. For the CT-based method, postoperative pre-digital (prior to 2000) and digital CT images were independently assessed in the axial, sagittal, and coronal planes, and residual gap and step displacement were measured along the articular surface at the level of the weight-bearing dome. Adequate reductions on postoperative CT were defined as $<1 \mathrm{~mm}$ step and $<5 \mathrm{~mm}$ gap displacement and inadequate reductions as $\geq 1 \mathrm{~mm}$ step and/or $\geq 5 \mathrm{~mm}$ gap displacement [12]. Assessment of postoperative PXR's and CT scans was performed by two observers $(* *, * *)$ and differences settled in consensus. The observers were blinded for clinical outcome and not involved in the initial surgical care of the included patients.

\section{Statistical analysis}

Statistical analysis was performed using SPSS version 24.0 (IBM Software, Armonk, NY, USA). Baseline characteristics of continuous variables were presented as means with standard deviation (SD) and ranges, and nominal data as number $(n)$ with percentage (\%). Two-by-two tables were constructed to show the relation between quality of reduction (adequate versus inadequate) as assessed according to the PXR and CT-based methods and native hip survivorship. Predictive values for both methods were compared using Pearson's Chi-square or Fisher's exact tests when appropriate. Kaplan-Meier curves were used to plot native hip survivorship for reduction quality based on both PXR and $\mathrm{CT}$ assessment, and log-rank tests were used to determine statistical differences. Cox regression analysis was used to 
identify dependent and independent risk factors associated with conversion to THA. Results are presented as hazard ratio's (HR) with 95\% confidence intervals $(95 \% \mathrm{CI})$. A $p$ value of $<0.05$ was considered to indicate a statistically significant result.

\section{Results}

\section{Baseline characteristics}

A total of 220 patients were included in this study. Mean age was 50.8 years (SD 17.8, range 18-91 years); 117 patients (53\%) were $\geq 50$ years of age and 142 patients (64\%) were male. The majority of patients had associated type fractures (64\%); (Table 1). Posterior wall impaction was found in 77 patients (18\%) and supero-medial dome impaction in 40 patients $(9 \%)$.

Mean follow-up was 8.9 years (SD 5.6, range $0.5-23.3$ years). A total of 52 patients had native hip failure and converted to THA (24\%) at a mean of 4.2 years (SD 4.2 range $0.3-21.0$ year); 27 patients (52\%) had early failure (within 2 years). Indication for conversion to THA was symptomatic osteoarthritis in 43 patients (83\%). A further five patients (10\%) had avascular necrosis of the femoral head, $3(6 \%)$ had fixation failure, and $1(2 \%)$ a deep infection; all of these patients had poor reductions on both PXR and CT.

\section{Quality of reduction}

An adequate reduction according to $\mathrm{CT}$ assessment (as compared to PXR) was associated with a higher predictive value for native hip survivorship (92\% versus $82 \%$; $p=0.043$ ); (Tables 2, 3). Inadequate reductions according

Table 1 Incidence of different fracture types in the total study cohort $(n=220)$

\begin{tabular}{lc}
\hline Acetabular fracture type & \\
\hline Elementary & $12(6 \%)$ \\
Anterior column & $3(1 \%)$ \\
Anterior wall & $0(0 \%)$ \\
Posterior column & $48(22 \%)$ \\
Posterior wall & $16(7 \%)$ \\
Transverse & \\
Associated & $8(4 \%)$ \\
T-shaped & $42(19 \%)$ \\
Transverse with posterior wall & $5(2 \%)$ \\
Posterior column with posterior wall & $46(21 \%)$ \\
Anterior column with posterior hemitransverse & $40(18 \%)$ \\
Both column & \\
\hline
\end{tabular}

Table 2 Quality of acetabular reduction, according to pelvic radiography (PXR) assessment versus native hip survivorship in the total cohort $(n=220)$

\begin{tabular}{llll}
\hline Total cohort & \multicolumn{3}{l}{ Hip survivorship } \\
\cline { 2 - 4 } & Yes & No & Total \\
\hline Reduction on PXR & & & \\
Adequate & $97(82 \%)$ & $21(18 \%)$ & $118(100 \%)$ \\
Inadequate & $71(70 \%)$ & $31(30 \%)$ & $102(100 \%)$ \\
Total & $168(76 \%)$ & $52(24 \%)$ & $220(100 \%)$ \\
\hline
\end{tabular}

to both methods were equally predictive for conversion to THA (33\% for CT and 30\% for PXR; $p=0.623$ ). Of 118 adequate PXR reductions, 61 (52\%) were graded inadequate on CT. Of these "missed" incongruencies 16 (26\%) converted to THA.

\section{Native hip survivorship}

Hip survivorship curves for reduction quality based on both PXR and CT assessment are shown in Fig. 1. For both methods, curves of adequate versus inadequate reductions were significantly different according to log-rank tests $(p=0.030$ for PXR and $p<0.001$ for CT). Ten-year native hip survivorship was higher for patients with an adequate versus inadequate reduction according to PXR assessment (79\%; 95\% CI $71-88 \%$ ) versus $68 \%$ (95\% CI $56-77 \%$ ) as well as based on CT assessment (89\%; 95\% CI 81-98\%) versus $65 \%$ (95\% CI 55-73\%).

Cox regression analysis identified the following (dependent) risk factors for conversion to THA; age $\geq 50$ years $(p<0.001)$, posterior wall $(p=0.002)$, and supero-medial dome impaction $(p<0.001)$ and inadequate reductions as assessed on PXR $(p=0.019)$ and CT $(p<0.001)$ (Table 4$)$. After adjusting for confounders, only age $\geq 50$ years $(p<0.001)$ and an inadequate reduction quality as assessed on CT ( $p=0.038)$ were found to be (independent) risk factors. Reduction quality as assessed on PXR was not found to be independently predictive for native hip failure $(p=0.585)$.

Table 3 Quality of acetabular reduction, according to CT-based assessment versus native hip survivorship in the total cohort $(n=220)$

\begin{tabular}{|c|c|c|c|}
\hline \multirow[t]{2}{*}{ Total cohort } & \multicolumn{3}{|c|}{ Hip survivorship } \\
\hline & Yes & No & Total \\
\hline \multicolumn{4}{|c|}{ Reduction on CT } \\
\hline Adequate & $77(92 \%)$ & $7(8 \%)$ & $84(100 \%)$ \\
\hline Inadequate & $91(67 \%)$ & $45(33 \%)$ & $136(100 \%)$ \\
\hline Total & $168(76 \%)$ & $52(24 \%)$ & $220(100 \%)$ \\
\hline
\end{tabular}


Fig. 1 Native hip survivorship curves for reduction quality based on both PXR and CT assessment

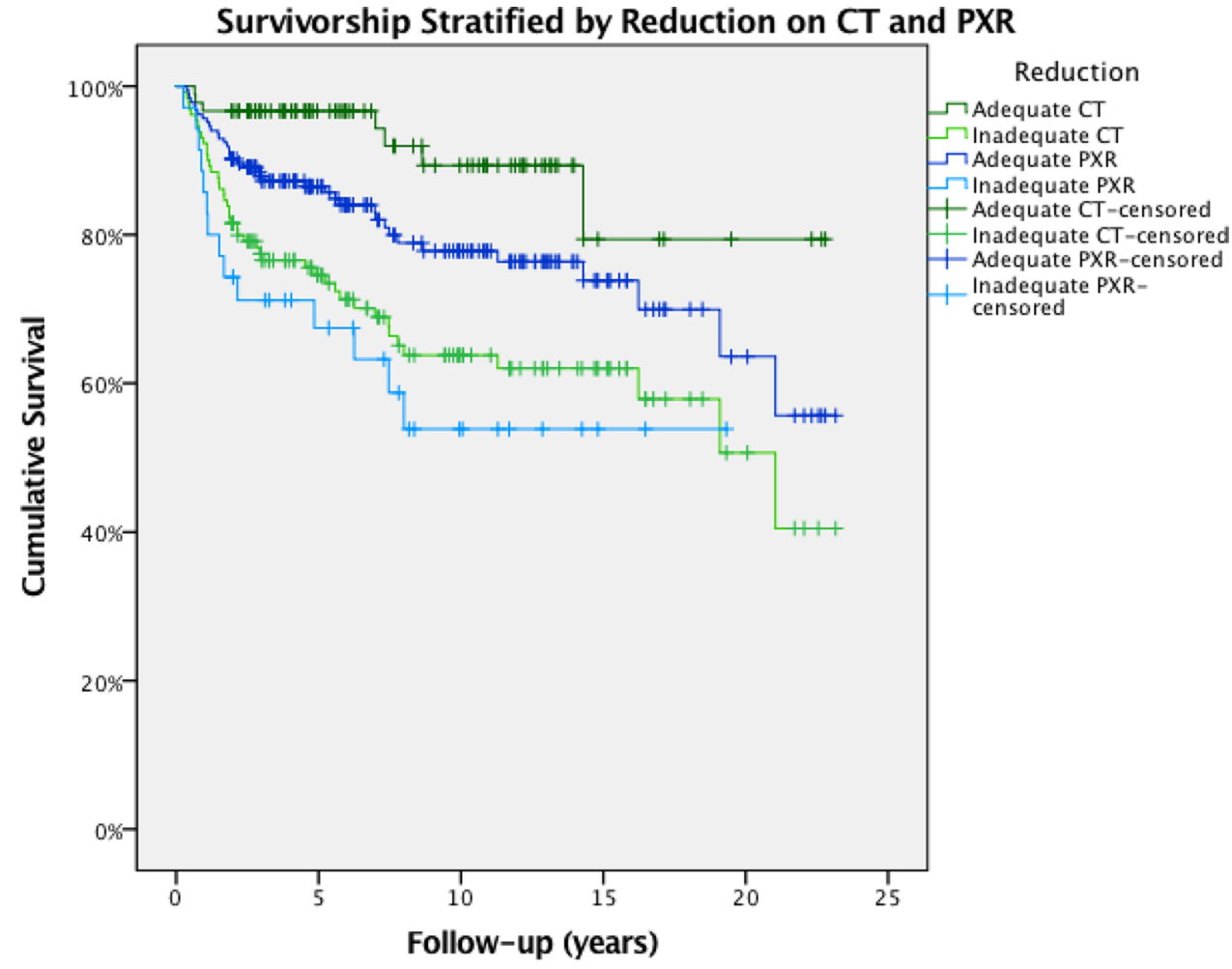

Table 4 Cox regression analysis for (dependent and independent) risk factors associated with conversion to total hip arthroplasty in all patients $(n=220)$

\begin{tabular}{|c|c|c|c|c|}
\hline & \multicolumn{2}{|l|}{ Unadjusted } & \multicolumn{2}{|l|}{ Adjusted } \\
\hline & HR $(95 \%$ CI $)$ & $p$ value & HR $(95 \%$ CI $)$ & $p$ value \\
\hline Age $(\geq 50$ years $)$ & $5.3(2.6-11.1)$ & $<0.001$ & $4.6(2.2-9.6)$ & $<0.001$ \\
\hline Gender (female) & $1.5(0.9-2.6)$ & 0.140 & $1.5(0.9-2.7)$ & 0.129 \\
\hline Fracture type (Associated) & $1.5(0.8-2.8)$ & 0.166 & $0.9(0.5-1.7)$ & 0.730 \\
\hline Posterior wall impaction & $2.4(1.4-4.1)$ & 0.002 & $1.7(0.9-3.0)$ & 0.081 \\
\hline Supero-medial dome impaction & $2.9(1.7-5.2)$ & $<0.001$ & $1.7(0.9-3.2)$ & 0.136 \\
\hline Reduction on PXR (inadequate) & $1.8(1.1-3.2)$ & 0.033 & $1.2(0.6-2.3)$ & 0.585 \\
\hline Reduction on CT (inadequate) & $4.1(1.9-9.2)$ & $<0.001$ & $2.6(1.1-6.2)$ & 0.038 \\
\hline
\end{tabular}

$P X R$ plain pelvic radiography

\section{Discussion}

Results of this study indicate that following ORIF of acetabular fractures, an adequate reduction according to CT-based assessment is more predictive for native hip survivorship than PXR-based assessment.

The improved performance of CT in this regard is likely due to its ability to more accurately detect even minor incongruencies within the acetabular joint surface. The previous studies have shown definitively that both pre- and post-operative $\mathrm{CT}$ is a superior modality for evaluation of acetabular fracture displacement $[9,10,11,13]$. The current study does not attempt to replicate these findings, but logically follows earlier work. In a prior study, PXR versus
CT assessment of postoperative acetabular reduction was compared using the Matta criteria [11]. Results showed that $75 \%$ of anatomic reductions $(0-1 \mathrm{~mm}$ displacement $)$ on PXR were classified imperfect or poor $(\geq 2 \mathrm{~mm}$ displacement) on CT. The question whether superior accuracy would result in better performance for CT in terms of predicting hip survivorship remained unanswered as it could be argued that more clinically irrelevant displacements may be detected using this modality. Based on our current results, it appears that at least some malreductions, which go undetected on PXR, can lead to symptomatic osteoarthritis ultimately necessitating THA conversion. These "missed" incongruencies may be of importance at longterm follow-up particularly in more active younger adults. 
In the current study, we chose to grade postoperative reductions on CT based on a system specifically designed for this modality (as the commonly used Matta criteria for reduction quality on PXR have not been validated for postoperative CT assessment [12]. For analysis of PXR results, adequate (or anatomic) reductions were compared to inadequate (or non-anatomic) reductions, similar to earlier studies $[2,3]$.

Our results further show that contrary to prediction of hip survivorship, prediction of hip failure based primarily on reduction quality (on PXR or CT) remains problematic. An inadequate reduction as assessed on both modalities was only predictive for THA conversion to a certain extent (31-35\%). Apparently, many patients are able to function reasonably well despite an inadequate acetabular reduction, perhaps by taking pain medication or by adaptation in terms of avoiding certain activities or lowering their activity level. Specifically in (less active or low-demand) elderly patients, inadequate acetabular reductions have previously shown limited correlation to functional outcome [14]. It is evident that, apart from a poor reduction, many (known and unknown) factors ultimately influence native hip failure and conversion to THA [1-3, 15-18].

While quality of reduction (as evaluated on both postoperative CT as well as PXR) showed an association with native hip survivorship, only an inadequate reduction as assessed on CT was identified as an independent risk factor for conversion to THA. After adjusting for (stronger) confounders, reduction quality based on PXR assessment was no longer found to be a significant risk factor in our patient cohort.

Several studies have sought to identify predictors for clinical or functional outcomes following acetabular fracture surgery $[4,6,7,19]$. However, a few studies have specifically examined the independent association between quality of reduction and native hip survivorship, and results have been inconclusive [1-3]. In the largest series of 810 patients, a non-anatomical (inadequate) reduction was found to be an independent risk factor for early conversion to THA (within 2 years); [3]. In a study of 285 patients examining long-term hip survivorship, reduction quality was not found to be an independent predictor in older and younger subgroups but only reached significance when measured as step deformity $(>2 \mathrm{~mm}$ ) on the obturator oblique radiograph in the total cohort [1]. In a further recent study with long-term followup, accuracy of reduction was not found to be a risk factor for a composite outcome (including conversion to THA) in acetabular fracture patients who underwent surgical hip dislocation [2]. Importantly, these prior studies all based their assessment of reduction quality on post-operative PXR rather than $\mathrm{CT}$ imaging.

Based on our findings, we propose that postoperative CTbased assessment of reduction quality could play a valuable role (particularly) in acetabular fracture research both for reliable evaluation of direct postoperative results as well as for prognostication purposes, for instance following the introduction of novel approaches or surgical implants. In addition, this modality may provide important information to improve the individual surgeon's technique. In our personal experience and in that of others, CT imaging following acetabular fracture surgery rarely results in re-intervention for misplaced hardware or malreduction [20]. However, in the future, the introduction of intra-operative CT scanning may allow direct revisions based on CT findings. Ultimately, benefits of postoperative $\mathrm{CT}$ assessment should be weighed against its apparent drawbacks in terms of radiation risk and increased costs.

Limitations of this study include the large proportion of patients excluded based on the absence of a complete set of postoperative imaging or an inadequate follow-up duration. Nevertheless, it should be considered that this study was designed to directly compare the performance of two methods within the same cohort of patients. In terms of external validity, it appears that our final patient cohort is largely comparable to earlier series of operatively treated acetabular fracture patients. Gender and fracture types were similar $[1,3,4,8]$, but overall conversion rate to THA $(24 \%)$ was somewhat higher than reported in earlier long-term followup studies (14-16\%) [1, 3, 4], in part related to our mean patient age (51 years), which was at the higher end of the spectrum as compared to prior study cohorts (ranging from $36-53$ years) $[1,4,20,21]$.

\section{Conclusion}

Following acetabular fracture surgery, native hip survivorship is better predicted using CT imaging as compared to PXR assessment. Predicting hip failure and need for THA in patients with inadequate reductions based on both assessment methods remain challenging. While both PXR and CTbased methods are associated with hip survivorship, only an inadequate reduction according to $\mathrm{CT}$ assessment was an independent risk factor for conversion to THA. As such CT could present a valuable tool particularly in future research.

Funding There is no funding source.

\section{Compliance with ethical standards}

Conflict of interest Each author certifies that he has no commercial associations (e.g., consultancies, stock ownership, equity interest, patent/licensing arrangements, etc.) that might pose a conflict of interest with the submitted article. This manuscript is an original work that has never been published previously. It has been reviewed by all of the above authors. 
Ethical approval This article does not contain any studies with human participants or animals performed by any of the authors.

Open Access This article is distributed under the terms of the Creative Commons Attribution 4.0 International License (http://creativecommons.org/licenses/by/4.0/), which permits unrestricted use, distribution, and reproduction in any medium, provided you give appropriate credit to the original author(s) and the source, provide a link to the Creative Commons license, and indicate if changes were made.

\section{References}

1. Clarke-Jenssen J, Roise O, Storeggen SAO, Madsen JE (2017) Long-term survival and risk factors for failure of the native hip joint after operatively treated displaced acetabular fractures. Bone Jt J 99(6):834-840

2. Haefeli PC, Marecek GS, Keel MJ, Siebenrock KA, Tannast M (2017) Patients undergoing surgical hip dislocation for the treatment of acetabular fractures show favourable long-term outcome. Bone Jt J 99-b(4):508-515. https://doi. org/10.1302/0301-620x.99b4.37681

3. Tannast M, Najibi S, Matta JM (2012) Two to twenty-year survivorship of the hip in 810 patients with operatively treated acetabular fractures. J Bone Jt Surg Am 94(17):1559-1567

4. Briffa N, Pearce R, Hill AM, Bircher M (2011) Outcomes of acetabular fracture fixation with ten years' follow-up. J Bone Jt Surg Br 93(2):229-236. https://doi. org/10.1302/0301-620X.93B2.24056

5. Giannoudis PV, Nikolaou VS, Kheir E, Mehta S, Stengel D, Roberts CS (2009) Factors determining quality of life and level of sporting activity after internal fixation of an isolated acetabular fracture. J Bone Jt Surg Br 91(10):1354-1359

6. Matta JM (1996) Fractures of the acetabulum: accuracy of reduction and clinical results in patients managed operatively within three weeks after the injury. J Bone Jt Surg Am 78(11):1632-1645

7. Moed BR, WillsonCarr SE, Watson JT (2002) Results of operative treatment of fractures of the posterior wall of the acetabulum. J Bone Jt Surg Am 84-A(5):752-758

8. Letournel E Jr (1993) Fractures of the acetabulum. In: Elson RA (ed) Translation of Les fractures du cotyle, 2nd edn. Springer, New York

9. Borrelli J Jr, Ricci WM, Steger-May K, Totty WG, Goldfarb C (2005) Postoperative radiographic assessment of acetabular fractures: a comparison of plain radiographs and CT scans. J Orthop Trauma 19(5):299-304

10. Moed BR, Carr SE, Gruson KI, Watson JT, Craig JG (2003) Computed tomographic assessment of fractures of the posterior wall of the acetabulum after operative treatment. J Bone Jt Surg Am 85-A(3):512-522

11. Verbeek DO, van der List JP, Villa JC, Wellman DS, Helfet DL (2017) Postoperative CT is superior for acetabular fracture reduction assessment and reliably predicts hip survivorship. J Bone Jt Surg Am 99(20):1745-1752. https://doi.org/10.2106/ JBJS.16.01446

12. Verbeek DO, van der List JP, Tissue CM, Helfet DL (2018) Predictors for long-term hip survivorship following acetabular fracture surgery: importance of gap compared with step displacement. J Bone Jt Surg Am 100(11):922-929. https://doi.org/10.2106/ JBJS.17.00692

13. Borrelli J Jr, Goldfarb C, Ricci W, Wagner JM, Engsberg JR (2002) Functional outcome after isolated acetabular fractures. J Orthop Trauma 16(2):73-81

14. Miller AN, Prasarn ML, Lorich DG, Helfet DL (2010) The radiological evaluation of acetabular fractures in the elderly. J Bone Jt Surg Br 92(4):560-564

15. Carroll EA, Huber FG, Goldman AT, Virkus WW, Pagenkopf E, Lorich DG, Helfet DL (2010) Treatment of acetabular fractures in an older population. J Orthop Trauma 24(10):637-644. https:// doi.org/10.1097/BOT.0b013e3181ceb685

16. Helfet DL, Borrelli J Jr, DiPasquale T, Sanders R (1992) Stabilization of acetabular fractures in elderly patients. J Bone Jt Surg Am 74(5):753-765

17. Helfet DL, Schmeling GJ (1994) Management of complex acetabular fractures through single nonextensile exposures. Clin Orthop Relat Res 305:58-68

18. Jeffcoat DM, Carroll EA, Huber FG, Goldman AT, Miller AN, Lorich DG, Helfet DL (2012) Operative treatment of acetabular fractures in an older population through a limited ilioinguinal approach. J Orthop Trauma 26(5):284-289

19. Giannoudis PV, Grotz MR, Papakostidis C, Dinopoulos H (2005) Operative treatment of displaced fractures of the acetabulum. A meta-analysis. J Bone Jt Surg Br 87(1):2-9

20. Archdeacon MT, Dailey SK (2015) Efficacy of routine postoperative CT scan after open reduction and internal fixation of the acetabulum. J Orthop Trauma 29(8):354-358

21. Ochs BG, Marintschev I, Hoyer H, Rolauffs B, Culemann U, Pohlemann T, Stuby FM (2010) Changes in the treatment of acetabular fractures over 15 years: analysis of 1266 cases treated by the German Pelvic Multicentre Study Group (DAO/DGU). Injury 41(8):839-851. https://doi.org/10.1016/j.injury.2010.04.010

Publisher's Note Springer Nature remains neutral with regard to jurisdictional claims in published maps and institutional affiliations. 